\title{
Computational fluid dynamics analysis of the effect of simulated plaques in the left coronary artery: A preliminary study
}

\author{
T. Chaichana ${ }^{\text {a }}$, J. Jewkes ${ }^{\mathrm{b}}$ and Z. Sun ${ }^{\mathrm{a}}$ \\ ${ }^{a}$ Discipline of Medical Imaging, Department of Imaging and Applied Physics, \\ Curtin University, Western Australia \\ ${ }^{b}$ Fluid Dynamics Research Group, Department of Mechanical Engineering, \\ Curtin University, Western Australia \\ Email:j.jewkes@,curtin.edu.au
}

\begin{abstract}
:
Background: Atherosclerosis is the most common cause of coronary artery disease which is formed by plaque presence inside the artery wall leading to blockage of the blood supply to the heart muscle. The mechanism of atherosclerotic development is dependent on the blood flow variations in the artery wall during cardiac cycles. Characterization of plaque components and investigation of the plaques with subsequent coronary artery stenosis and myocardial dysfunction has been extensively studied in the literature. However, little is known about the effect of plaques on hemodynamic changes to the coronary artery, to the best of our knowledge. Investigation of the position of plaques in the coronary artery and its corresponding regional hemodynamic effects will provide valuable information for prediction of the coronary artery disease progression. The aim of this study is to investigate the effect of simulated plaques in the left coronary artery using computational fluid dynamics.
\end{abstract}

Methods: A left coronary artery model was generated based on a computed tomography data in a patient suspected of coronary artery disease. The model consists of the left main coronary artery, left anterior descending and left circumflex, together with side branches. Simulated coronary plaques were created and placed in the left main coronary artery and left anterior descending with a resultant lumen stenosis of more than 50\%. The blood rheology and pulsatile velocity at the left coronary artery were applied to simulate the realistic physiological situation. A transient simulation was performed to demonstrate the hemodynamic changes during cardiac phases. The flow velocity pattern, wall shear stress and wall pressure were measured at peak systolic and middle diastolic phases in the models with and without presence of plaques.

Results: Our results showed that the flow change due to the simulated coronary plaques demonstrated a large circulation region at the left coronary bifurcation, and the velocity through bifurcation was increased. In contrast, a smooth flow pattern was observed in the non-calcified regions and flow velocity was low at the bifurcation. Low wall pressure was present in the coronary artery with a simulated coronary plaque whereas there was high wall pressure in the normal coronary artery. The simulated plaques resulted in high wall shear stress when compared to the low wall shear stress present in the normal coronary artery. The simulated coronary plaques interfered with blood flow behavior which was demonstrated as a large region of disturbed flow at coronary bifurcation.

Conclusion: We successfully simulated the coronary plaques in a realistic coronary model and the effect of plaques in different locations on subsequent hemodynamic changes. Our preliminary study is useful for further investigation of the development of atherosclerosis in patients with different cardiac risk factors.

Keywords: Coronary plaque, wall shear stress, wall pressure, blood flow simulation, computational fluid dynamic 


\section{INTRODUCTION}

Coronary artery disease (CAD) is commonly caused by the presence of plaques on the coronary wall. Plaques resulted in narrowing coronary lumen and associated with a blood clot compromised hemodynamic changes to the myocardium (Australian Institute of Health and Welfare, 2006). It is well known that coronary angulation is associated with the development of atherosclerotic plaques and coronary plaques were generally formed at the bifurcation area, as confirmed by previous studies (Askura and Karino 1990; Chaichana et al., 2011; Fuser, 1994; Gziut et al., 2006; Han et al., 2010; Sun et al., 2011). Blood flow changes are believed to play a role in the development of coronary plaques, and this is demonstrated by the change of hemodynamic parameters such as wall shear stress (WSS) and blood flow velocity (Askura and Karino 1990; Chaichana et al., 2011; Fuser, 1994). Therefore, the local flow alteration at the coronary bifurcation in realistic vascular geometry is important for understanding the pathogenesis of coronary disease.

Modern imaging techniques such as intravascular ultrasound (IVUS) and computed tomography (CT) virtual intravascular endoscopy (VIE) are used for assessment of CAD. However, early detection and diagnosis are of particularly important for the reduction of mortality and subsequent complications (Australian Institute of Health and Welfare, 2006). The IVUS is used to measure blood flow changes, but is restricted to the large coronary artery, and fails to assess WSS changes (Gijsen et al., 2007; Grayburn et al., 1992). The VIE has been used to visualize and assess coronary plaques in patients suspected of CAD (Sun et al., 2010), but is limited to the anatomical details and is unable to provide the hemodynamic changes in the coronary artery due to the presence of plaques. Computational fluid dynamics (CFD) is used to overcome those imaging techniques and prior studies used CFD to analyze the hemodynamic parameters in a reconstructed coronary artery to implicate plaques progression (Farmakis et al., 2004; Giannoglou et al., 2005; Johnston et al., 2004; Katritsis et al., 2010; Perktold et al., 1998; Shanmugavelayudam et al., 2010; Soulis et al., 2006;). The purpose of this study is to simulate plaques in the left coronary artery and investigate the corresponding influence of plaques on hemodynamic variation at the coronary bifurcation.

\section{MATERIAL AND METHODS}

\subsection{Left Coronary Reconstruction Procedure}

A sample patient suspected of coronary artery disease was selected for inclusion in the study. The patient data was obtained using a multislice CT scanner with patient's CT volume data saved in 'DICOM format' for three-dimensional reconstruction. Commercially available software, Analyze 7.0 (Analyze Direct, Inc., Lexana, KS, USA) was used to segment the left coronary artery section and its coronary tree. Image post processing and editing techniques were applied to the volume data, and digital artifacts and soft tissues were manually removed in some slices (Sun et al., 2003; Sun et al., 2004). Figure 1 shows the three-dimensional

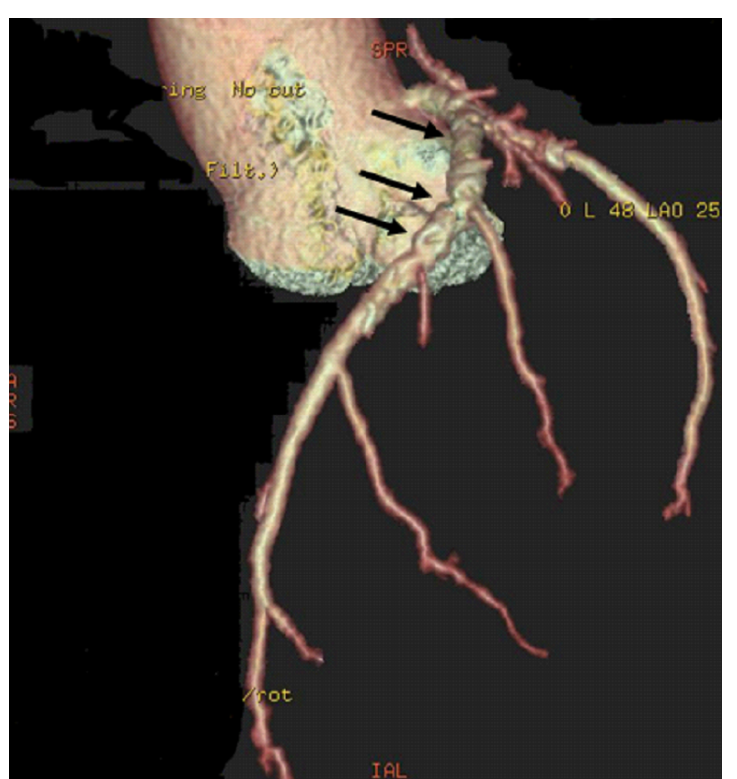

Figure 1.3D volume rendering demonstrates stenotic changes in the LAD (arrows). CT image of the left coronary artery. The left coronary model consisted of the left main stem (LMS), left anterior descending (LAD), left circumflex (LCx) and its coronary tree. The reconstruction procedure carried out using Blender version 2.48 (Blender Institute, Amsterdam, Netherlands). The computer models were saved in 'STL format' for hex-mesh generation.

\subsection{Left Coronary models With/Without Plaques}

Coronary plaques were generated with a $50 \%$ lumen stenosis leading to major blood flow variations inside the coronary artery (Fuser, 1994). However, plaques contain various components that are determined by their calcium concentrations (Sun et al., 2010). The hard plaques or central calcified plaques were simulated in this study and located at LMS and LAD, as this type of plaque represents the most common form of $\mathrm{CAD}$, and plaques located in these locations 
tend to induce myocardial ischemic changes (Cho et al., 2001). Figure 2 shows the left coronary artery models with and without presence of coronary plaques.

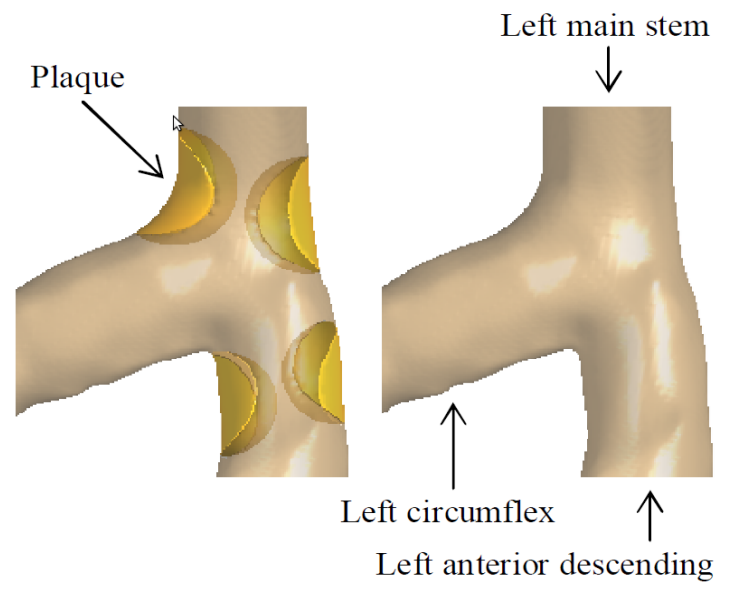

Figure 2. 3D left coronary artery models with and without plaques.

\subsection{Computational Fluid Dynamics}

The STL files in Section 1.2 were used to generate the mesh models, using ANSYS ICEM CFD 12 (Ansys, Inc., Canonsburg, PA, USA). The mesh elements of the left coronary models, with and without plaques, were created using a hex mesh of approximately $1 \times 10^{6}$ of elements. The plaque section was created using a tetra mesh of appproximately $8 \times 10^{4}$ cells. The materials and fluid properties for computational simulation were referenced from a previous study (Chaichana et al., 2011). In this study, time-dependent boundary conditions were applied to the velocity inlet (left main stem) and the pressure outlets (left anterior descending and left circumflex). Plaques were assumed to be a rigid (Sun et al., 2009). Threedimensional left coronary mesh models were saved in 'GTM format' and CFD analysis has been described in previous studies (Chaichana et al., 2011; Sun and Chaichana, 2010; Sun and Chaichana, 2009).

The Navier-Stokes equations were solved by using ANSYS CFX 12 (Ansys, Inc., Canonsburg, PA, USA), running on Microsoft Windows 7, 6 MB RAM with a Xeon W3505 2.53 GHz CPU. The convergence target of each simulation was less than $1 \times 10^{-4}$ per left coronary model. Time consumption of each model was approximately 2 hours. ANSYS CFD Post-processing 12 (Ansys, Inc., Canonsburg, PA, USA) was used to compute and visualize velocity flow fields for comparisons of left coronary models, with and without presence of plaques.

\section{RESULTS}

\subsection{Hemodynamic variations surrounding plaques locations}

The present study was performed based on in-vivo physiological conditions during the cardiac pulsatile cycle. The time of $0.4 \mathrm{sec}$ and $0.7 \mathrm{sec}$ indicate the peak systolic and mid diastolic phases, respectively. The results of computer simulations demonstrated a direct influence of bifurcation plaques at the left coronary artery on blood flow variations. Figure 3 presents the flow velocity patterns visualized at the bifurcation without the presence of plaques. While the blood flow surrounding plaque locations is shown in Figure 4. The velocity contours range from $0 \mathrm{~mm} / \mathrm{s}$ to $30.50 \mathrm{~mm} / \mathrm{s}$, as shown in Figure 4 . The left coronary model with plaques caused hemodynamic variations and flow velocity results increased greatly in the presence of plaques. Peak velocity ranged from $28.32-30.50 \mathrm{~mm} / \mathrm{s}$, at the plaque locations. Recirculating regions were found at postplaque locations in the LMS, LAD and LCx (Figure 4). In contrast, Figure 3 displayed that the smooth velocity flow pattern which was observed in the left coronary model without plaques and high velocity ranged from 21.79-23.96 mm/s (peak systolic) and 28.32-30.50 mm/s (mid diastolic, not shown) at the bifurcation location.

\section{DISCUSSION}

This study shows that the coronary plaques in the left coronary artery result in blood flow variations when compared with the left coronary model without presence of plaques. This is clinically significant as these blood flow changes may induce further atherosclerotic changes in the coronary artery side branches, thus leading to potential damage to the coronary wall. 


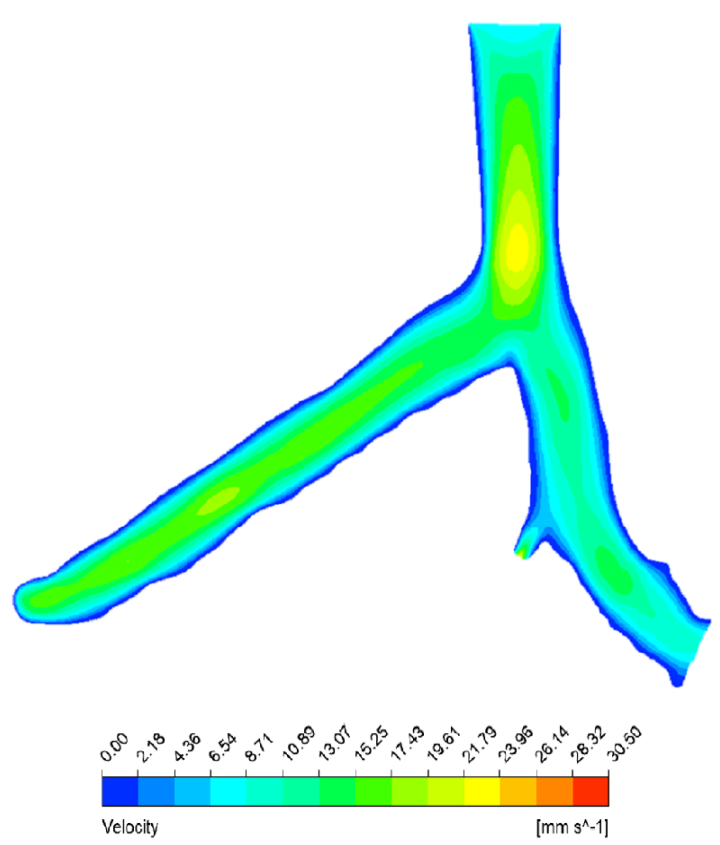

Figure 3. Flow velocity observed in left coronary model without presence of plaque during systolic peak of $0.4 \mathrm{~s}$. This presents the normal condition of flow field at left coronary bifurcation.

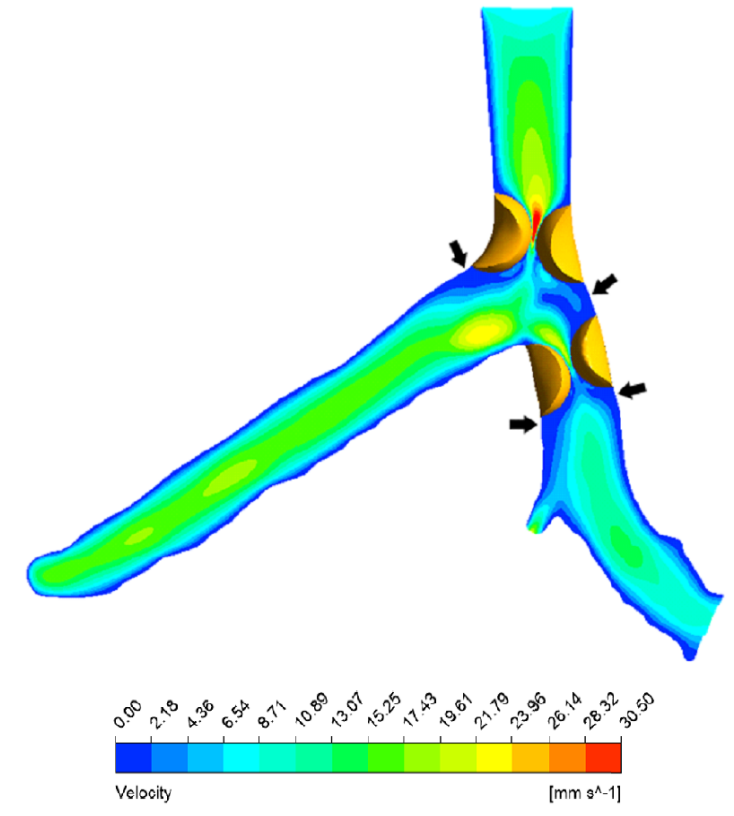

Figure 4. Flow velocity observed in left coronary model with presence of plaque during systolic peak of $0.4 \mathrm{~s}$. Arrows reveal the locations of low flow velocity which occurred at post-plaque positions

Plaques are commonly formed in the coronary bifurcation due to coronary angulations, resulting in local low wall shear stress, as proved by our and other studies (Askura and Karino 1990; Chaichana et al., 2011; Fuser, 1994; Gziut et al., 2006; Han et al., 2010; Sun et al., 2011). Medical image visualisation simply provides morphological changes in the coronary artery. These techniques have been used to identify and detect location of coronary plaques and degree of lumen stenosis (Gijsen et al., 2007; Grayburn et al., 1992; Sun et al., 2010). In contrast, CFD offers hemodynamic analysis caused by the presence of plaques at the left coronary bifurcation (Chaichana et al., 2011; Sun and Chaichana, 2010; Sun and Chaichana 2009). Therefore, the influence of plaques in the left coronary artery could be well understood with regard to hemodynamic changes, and it is beyond the imaging analysis of coronary stenosis or occlusion.

It is well known that the velocity is a standard factor related to the WSS alterations, as low WSS regions represent the locations where the flow velocity is low (Askura and Karino 1990; Chaichana et al., 2011; Fuser, 1994). Our CFD analysis of the left coronary model with and without plaques demonstrated that there is direct relationship between bifurcation plaques and blood flow changes, as flow velocity was low at postplaques locations and this could be lead to low WSS at the same positions (Figure 4). In contrast, the normal flow condition is shown in Figure 3 and the high velocity appeared at bifurcation at the above position of merging between LAD and LCX.

There are some limitations in this study that should be addressed. Firstly, the different positions of realistic coronary plaques may affect the flow field changes. Secondly, realistic left coronary models with and without plaques were assumed to have a rigid wall rather than an elastic wall. Thirdly, the blood viscosity was assumed to be Newtonian. This assumption is acceptable as confirmed by a previous study (Johnston et al., 2004). Further studies will use coronary artery models with a more realistic idealized geometry expanded to estimate the different plaque positions. 


\section{CONCLUSION}

In this study, we investigated the influence of plaques in realistic left coronary artery models, on blood flow variations at coronary bifurcation with use of left coronary models, with and without the presence of plaques. The direct correlation on blood flow changes was found in the location where the plaques were present and the low flow velocity regions were found at after the plaques location. This indicates that plaques tend to form in the low velocity areas. Our results enhance the understanding of coronary artery disease with the presence of plaques. Future studies will focus on the various plaque positions in the left coronary artery, and characterize the hemodynamic parameters required to verify our results.

\section{REFERENCES}

Asakura, T., and Karino, T. (1990). Flow patterns and spatial distribution of atherosclerotic lesions in human coronary arteries. Circulation Research, 66, 1045-66.

Australian Institute of Health and Welfare. (2006). The tenth biennial health report of the Australian Institute of Health and Welfare. Cat. no. AUS 73. Canberra: AIHW.

Chaichana, T., Sun, Z., and Jewkes, J. (2011). Computation of haemodynamics in the left coronary artery with variable angulations. Journal of Biomechanics, 44, 1869-1878.

Cho, G.Y., Lee, C.W., Hong, M.K., Kim, J.J., Park, S.W., and Park, S.J. (2001). Effects of stent desing on side branch occlusion after coronary stent placement. Catheterization and Cardiovascular Interventions, 52, 18-23.

Farmakis, T.M., Soulis, J.V., Giannoglou, G.D., Zioupos, G.J., and Louridas, G.E. (2004). Wall shear stress gradient topography in the normal left coronary arterial tree: possible implications for atherogenesis. Current medical research and opinion, 5, 587-96.

Fuser, V. (1994). Lewis A. Conner memorial lecture. Mechanisms to myocardial infarction: insights from studies of vascular biology. Circulation, 90, 2126-46.

Giannoglou, G.D., Soulis, J.V., Farmakis, T.M., Giannakoulas, G.A, Parcharidisa, G.E., and Louridas, G.E. (2005). Wall pressure gradient in normal left coronary artery tree. Medical Engineering and Physics, 27, 455-64.

Gijsen, F.J.H., Wentzel, J.J., Thury, A., Lamers, B., Schuurbiers J.C.H., Serruys, P.W., and van der Steen, A.F. (2007). A new imaging technique to study 3-D plaque and shear stress distribution in human coronary artery bifurcations in vivo. Journal of Biomechanics, 40, 2349-57.

Grayburn, P.A., Willard, J.E., Haagen, D.R., Brickner, M.E., Alvarez, L.G., and Eichhorn, E.J. (1992). Measurement of coronary flow using high-frequency intravascular ultrasound imaging and pulsed Doppler velocimetry: in vitro feasibility studies. Journal of the American Society of Echocardiography, 5, 5-12.

Gziut, A.I. (2006). Comparative analysis of atherosclerotic plaque distribution in the left main coronary artery and proximal segments of left anterior descending and left circumflex arteries in patients qualified for percutaneous coronary angioplasty. Annales Academiae Medicae Stetinensis, 52, 51-62.

Han, S.H., Puma, J., Garcia-Garcia, H.M., Nasu, K., Margolis, P., Leon M.B., and Lerman, A. (2010) Tissue characterisation of atherosclerotic plaque in coronary artery bifurcations: an intravascular ultrasound radiofrequency data analysis in humans. EuroIntervention, 6, 313-20.

Johnston, B.M., Johnston, P.R., Corney, S., and Kilpatrick, D. (2004). Non-Newtonian blood flow in human right coronary arteries: steady state simulations. Journal of Biomechanics, 37, 709-20.

Katritsis, D.G., Theodorakakos, A., Pantos, I., Andriotis, A., Efstathopoulos, E.P., Siontis, G., Karcanias, N., Redwood, S., and Gavaises, M. (2010). Vortex formation and recirculation zones in left anterior descending artery stenosis: computational fluid dynamics analysis. Physics in Medicine and Biology, 55, 1395-411.

Perktold, K., Hofer, M., Rappitsch, G., Loew, M., Kuban, B.D., and Friedman, M.H. (1998). Validated computation of physiologic flow in a realistic coronary artery branch. Journal of Biomechanics, 31, 21728.

Shanmugavelayudam, S.K., Rubenstein, D.A., and Yin, W. (2010). Effect of geometrical assumptions on numerical modelling of coronary blood flow under normal and disease conditions. Journal of Biomechanical Engineering, 132, 061004.

Soulis, J.V., Farmakis, T.M., Giannoglou, G.D., and Louridas, G.E. (2006). Wall shear stress in normal left coronary artery tree. . Journal of Biomechanics 39, 742-743.

Sun, Z., Winder, R.J., Kelly, B.E., Ellis, P.K., and Hirst, D.G. (2003). CT virtual intravascular endoscopy of abdominal aortic aneurysms treated with suprarenal endovascular stent grafting. Abdominal Imaging, 28, 580-7.

Sun, Z., Winder, R.J., Kelly, B.E., Ellis, P.K., Kennedy, P.T., and Hirst, D.G. (2004). Diagnostic value of CT virtual intravascular endoscopy in aortic stent grafting. Journal of Endovascular Therapy, 11, 13-25. 
Sun, Z., and Chaichana, T. (2009). Investigation of the hemodynamic effect of stent wires on renal arteries in patients with abdominal aortic aneurysms treated with suprarenal stent-grafts. CardioVascular and Interventional Radiology, 32, 647-57.

Sun, Z., Mwilpatayi, B., Chaichana, T., and Ng, C. (2009). Hemodynamic effect of calcified plaque on blood flow in carotid artery disease: a preliminary study. $3^{\text {rd }}$ International Conference on Bioinformatics and Biomedical Engineering, Beijing, 11 - 13 June 2009, 2009. Conference CD.

Sun, Z., and Chaichana, T. (2010). Fenestrated stent graft repair of abdominal aortic aneurysm: hemodynamic analysis of the effect of fenestrated stents on the renal arteries. Korean Journal of Radiology, 11, 95-106.

Sun, Z., and Cao, Y. (2011). Multislice CT angiography assessment of left coronary artery: Correlation between bifurcation angle and dimensions and development of coronary artery disease. European Journal of Radiology, published online 2 May 2011, doi:10.1016/j.ejrad.2011.04.015.

Sun, Z., Dimpudus, F.J., Nugroho, J., and Adipranoto, J.D. (2010). CT virtual intravascular endoscopy assessment of coronary artery plaques: A preliminary study. European Journal of Radiology, 75, e112-19. 